\title{
Correction to: Clinical-scale production of Aspergillus-specific T cells for the treatment of invasive aspergillosis in the immunocompromised host
}

\author{
Anastasia Papadopoulou ${ }^{1} \cdot$ Maria Alvanou $^{1,2} \cdot$ Kiriakos Koukoulias $^{1,2} \cdot$ Evangelia Athanasiou $^{1} \cdot$ \\ Andriana Lazaridou ${ }^{1} \cdot$ Nikolaos Savvopoulos $^{1,2} \cdot$ Panayotis Kaloyannidis $^{3}$ - Anthi-Marina Markantonatou ${ }^{4}$.

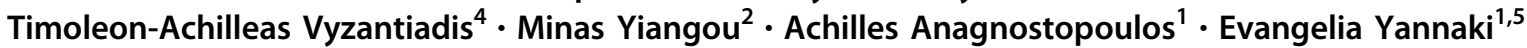

Published online: 20 June 2019

(c) Springer Nature Limited 2019

\section{Correction to: Bone Marrow Transplantation} https://doi.org/10.1038/s41409-019-0501-9

Published online 13 March 2019

The following funding statement was missing from the original article:
"Funding for this project was provided in part by an advanced EHA (European Hematology Association) Research Grant award and by a fellowship of excellence for postdoctoral studies from the Greek State Scholarships Foundation (IKY, SIEMENS program)"

This has been updated in the Acknowledgements section of the original article.

Anastasia Papadopoulou

apapadopoulou.gpapanikolaou@n3.syzefxis.gov.gr

1 Hematology Department-Hematopoietic Cell Transplantation

Unit, Gene and Cell Therapy Center, "George Papanikolaou"

Hospital, Thessaloniki 57010, Greece

2 Department of Genetics, Development and Molecular Biology, School of Biology, Aristotle University of Thessaloniki, Thessaloniki 54124, Greece

3 Adult Hematology and Stem Cell Transplant, King Fahad Specialist Hospital Dammam, Dammam 32253, Saudi Arabia

4 1st Department of Microbiology, Medical School, Aristotle University of Thessaloniki, Thessaloniki 54124, Greece

5 Department of Medicine, University of Washington, Seattle, WA 98195, USA 